\title{
Hyaluronic acid-coated nanoemulsions loaded with a hydrophobic ion pair of all-trans retinoic acid for improving the anticancer activity
}

\author{
Letícia Márcia da Silva Tinocoํㅜ, Flávia Lidiane Oliveira da Silva ${ }^{1}$, Lucas Antônio Miranda \\ Ferreira $^{2}$, Elaine Amaral Leite ${ }^{2}$, Guilherme Carneiro ${ }^{1 *}$
}

\begin{abstract}
${ }^{1}$ Department of Pharmacy, Faculty of Biological and Health Sciences, Federal University of Jequitinhonha and Mucuri Valleys, Diamantina, Minas Gerais, Brazil, ${ }^{2}$ Department of Pharmaceutics, Faculty of Pharmacy, Federal University of Minas Gerais, Belo Horizonte, Minas Gerais, Brazil
\end{abstract}

\begin{abstract}
All-trans retinoic acid (ATRA) has been studied for the treatment of cancer, including leukemia and breast cancer. This work aims to develop nanoemulsions (NE) loaded with a hydrophobic ion pair (HIP) of all-trans retinoic acid (ATRA) and a lipophilic amine, stearylamine (SA), and coated with hyaluronic acid (HA) to enhance anticancer activity and reducing toxicity. Blank NE was prepared by spontaneous emulsification and optimized prior to HIP incorporation. NE-ATRA was electrostatically coated with different concentrations of HA. Incorporation of ATRA-SA led to monodisperse NE with small size (129 $\pm 2 \mathrm{~nm}$; IP $0.18 \pm 0.005)$ and positive zeta potential $(35.7 \pm 1.0 \mathrm{mV})$. After coating with $0.5 \mathrm{mg} / \mathrm{mL} \mathrm{HA}$ solution, the mean diameter slightly increased to $158 \pm 5 \mathrm{~nm}$ and zeta potential became negative $(-19.7 \pm$ $1.2 \mathrm{mV}$ ). As expected, high encapsulation efficiency (near 100\%) was obtained, confirmed by polarized light microscopy and infrared analysis. Formulations remained stable over 60 days and release of ATRA from NE was delayed after the hydrophilic HA-coating. HA-coated NE-ATRA was more cytotoxic than free ATRA for MDA-MB-231 and MCF-7 breast cancer cell lines, especially in the CD44 overexpressing cells. Blank coated formulations showed no cytotoxicity. These findings suggest that this easily-made HA-coated NE-ATRA formulation is a promising alternative for parenteral administration, thus improving the breast cancer therapy with this drug.
\end{abstract}

Keywords: All-trans-retinoic acid. Breast neoplasms.Drug delivery. Hyaluronic acid. Nanoemulsion.

\section{INTRODUCTION}

All-trans retinoic acid (ATRA) is an important vitamin A derivative, which plays an anticancer role by binding to the nuclear receptors, retinoic acid receptors (RAR), and retinoid $\mathrm{X}$ receptors (RXR). ATRA regulates a variety of genes involved in cell proliferation and differentiation (Di Masi et al., 2015), and promotes cell proliferation inhibition and apoptosis by arresting the cell cycle in the G1 phase (Tang, Gudas, 2011). ATRA has been mainly used as a differentiation agent for treatment of leukemia, especially acute promyelocytic leukemia (Petrie, Zelent, Waxman, 2009), but is also effective

\footnotetext{
*Correspondence: G. Carneiro. Departamento de Farmácia, Faculdade de Ciências Biológicas e da Saúde, Universidade Federal dos Vales do Jequitinhonha e Mucuri. Rodovia MGT 367, Km 583, 39100-000 Diamantina, MG, Brazil. Phone: +5538 3532-1200* 8865 / FAX: +55 3835321249 . E-mail: guilherme. carneiro@ufvjm.edu.br / guifafar@yahoo.com.br.
}

against other types of cancer such as melanoma, liver, and breast carcinomas.

ATRA biodisponibility is low and quite variable when orally administered, which is associated with its low aqueous solubility $(\log \mathrm{P}=4.6,<1 \mathrm{mg} / \mathrm{mL}$ ) (Adamson et al., 1993; Abdulmajed, Heard, 2004). This has limited the choice of a proper solvent for intravenous administration of ATRA. On the other hand, prolonged oral treatment with ATRA leads to a progressive decrease in drug plasmatic concentrations due to induced metabolism via the cytochrome P450 system (Muindi et al., 1992). All these factors have limited the clinical use of both oral and intravenous ATRA formulations. An alternative to enabling the development of intravenous formulations has been the incorporation of ATRA in lipid nanostructured systems such as nanoemulsions (NE), solid lipid nanoparticles (SLN), nanostructured lipid carriers, or liposomes (Hwang et al., 2004; Lim, Lee, Kim 2004; Chansri et al., 2006; 
Kawakami et al., 2006; Carneiro et al., 2012; Silva et al., 2015).

Despite its lipophilicity, encapsulation of ATRA in lipid systems such as SLN is usually low (Jenning, Gohla, 2001) unless a high surfactant/lipid ratio is used (Lim, Kim, 2002; Hu, Tang, Cui 2004). Our group has previously reported that the in situ formation of hydrophobic ion pairs (HIP) of ATRA and lipophilic amines such as stearylamine (SA), benethamine, and maprotiline can significantly increase the ATRA incorporation within the lipid matrix (Castro et al., 2009; Carneiro et al., 2012; Silva et al., 2015; Silva et al., 2016).

Nanoparticles have been found to accumulate in tumor sites after intravenous administration due to the tumor architecture, which involves fenestrations in the endothelium and absence of effective lymphatic drainage (Torchilin, 2011). However, this enhanced permeation and retention effect does not assure tumor penetration by all types of nanosystems, so drugs can be released before the nanocarriers are taken up by the cancer cells, resulting in therapeutic inefficacy. Considering that the target of ATRA is the nuclear receptors, its intracellular delivery is important to attain an enhanced therapeutic effect for cancer.

Hyaluronic acid (HA) has been utilized in the active targeting of nanoparticles to tumors due to its ability to specifically bind to CD44 receptors (Mattheolabakis et al., 2015) overexpressed in some cancer cells. Therefore, the main advantages of coating nanoparticles with HA are the selective targeting and long blood circulation resulting from the hydrophilic surface comprising HA (Almalik, Day, Tirelli, 2013; Tran et al., 2014). Also, HA has the advantages of being biocompatible, biodegradable, and non-immunogenic, which makes it a good ligand to be used in the coating of nanocarriers for drug delivery applications (Rivkin et al., 2010; Sun et al., 2012). Thus, the main goal of this work was to develop an innovative formulation of NE loaded with ATRA and coated with HA as an alternative to improve the cancer therapy. The in situ formation of HIP was utilized to enhance ATRA encapsulation along the experimental design of the formulations. In addition, in vitro cytotoxicity was investigated against breast cancer cells.

\section{MATERIALS AND METHODS}

\section{Materials}

ATRA was kindly provided by BASF (Ludwigshafen, Germany); Super Refined Tween $80^{\mathrm{TM}}$ (Polysorbate 80 ) and Crodamol ${ }^{\mathrm{TM}}$ GTCC (medium chain triglycerides
[MCT]; caprylic/capric triglycerides) were provided by Croda Inc (Edison, NJ, USA). Stearylamine (SA; octadecylamine) and egg-lecithin (Lipoid ${ }^{\circledR}$ E80) were acquired from Sigma-Aldrich (St Louis, MO, USA) and Lipoid (Ludwigshafen, Germany), respectively. HA (MW $350 \mathrm{kDa}$ ) was purchased from All Chemistry (São Paulo, SP, Brazil).

For the in vitro studies, Dulbecco's modified Eagle's medium was obtained from Sigma-Aldrich (St Louis, MO, USA). Fetal bovine serum (FBS) and 3-(4,5-dimethylthiazol-2-yl)-2,5-diphenyl tetrazolium bromide (MTT) were purchased from Life Technologies (Carlsbad, CA, USA). The following cancer cell lines were used: MCF-7 (human breast adenocarcinoma from bone metastasis) and MDA-MB-231 (human breast adenocarcinoma, derived from metastatic site) cells were acquired from American Type Culture Collection (Manassas, VA, USA). All other chemicals were of analytical grade.

\section{Preparation and development of NE formulations}

NE was prepared by spontaneous emulsification (Bouchemal et al., 2004). Compositions of the formulations were based on previous studies (Castro et al., 2009; Carneiro et al., 2012), but with some modifications. Briefly (batch $10 \mathrm{ml}$ ), the oily phase (OP), including the organic solvent, and the aqueous phase (AP) were weighted and prepared separately. When present, ATRA was added in the OP. Next, the OP was added dropwise to the AP under vigorously magnetic stirring for $10 \mathrm{~min}$. The solvent was then removed by evaporation during $60 \mathrm{~min}$ under reduced pressure (Fisatom, São Paulo, Brasil). The $\mathrm{pH}$ of NE was adjusted to 7.0 with a $0.01 \mathrm{M} \mathrm{HCl}$ solution, using a $\mathrm{pH}-$ meter MS Tecnopon ${ }^{\circledR}$ (mPA 210, Piracicaba, Brazil).

HA coating was performed by electrostatic attraction (Yang et al., 2013). Different HA solutions were employed to select the ideal concentration for NE coating. In a typical procedure, $1 \mathrm{~mL}$ of NE dispersion was slowly added under vigorous magnetic stirring to a $\mathrm{HA}$ solution at concentrations of $0.01,0.25,0.50,1.0,2.0$, or $4.0 \mathrm{mg} / \mathrm{mL}$.

Optimization of the blank NE composition was performed by evaluating the influence of surfactant combination (Table I) and solvents on the physicochemical parameters of NE. Ethanol was utilized in NE 1-3, while acetone was used in NE 4. The inclusion of a sonication stage of $10 \mathrm{~min}$ with an ultrasound bath before the solvent removal was also evaluated (corresponding to NE 5).

After proper optimization of blank formulations, $0.05 \%$ ATRA was utilized to prepare ATRA-loaded NE 
Hyaluronic acid-coated nanoemulsions loaded with a hydrophobic ion pair of all-trans retinoic acid

TABLE I - Composition of NE formulations containing different surfactant systems

\begin{tabular}{|c|c|c|c|c|c|}
\hline Ingredient (\% w/v) & NE 1 & NE 2 & NE 3 & NE 4* & NE 5** \\
\hline \multicolumn{6}{|l|}{ Oily phase } \\
\hline MCT & 1.5 & 1.5 & 1.5 & 1.5 & 1.5 \\
\hline SA & 0.1 & 0.1 & 0.1 & 0.1 & 0.1 \\
\hline Egg lecithin & - & 0.5 & 0.5 & 0.5 & 0.5 \\
\hline Cholesterol & 0.15 & 0.15 & - & - & - \\
\hline \multicolumn{6}{|l|}{ Aqueous phase } \\
\hline Glycerol & 2.25 & 2.25 & 2.25 & 2.25 & 2.25 \\
\hline Tween $^{\circledR} 80$ & 1.0 & 0.5 & 0.5 & 0.5 & 0.5 \\
\hline Water q.s. & 100 & 100 & 100 & 100 & 100 \\
\hline
\end{tabular}

*NE 4 used acetone rather than ethanol as organic solvent; **NE 5 has the same composition of NE 4, but includes a 10 minutes sonication process previously the solvent removal.

(ATRA-NE). Finally, ATRA-NE was utilized in the HA coating studies performed by electrostatic attraction.

\section{Droplet size analysis and zeta potential}

The globule diameter of NE in the dispersion was determined by unimodal dynamic light scattering analysis. Data were reported as mean globule diameter, evaluated as the intensity obtained from three repeated measurements, and the polydispersity index. Zeta potential measurements were carried out by the dynamic light scattering associated with electrophoretic mobility, also in triplicate. All determinations were performed using a Zetasizer 3000 HSA (Malvern Instruments, Malvern, UK), at fixed angles of $90^{\circ}$ and $25^{\circ} \mathrm{C}$.

\section{Drug encapsulation efficiency}

Encapsulation efficiency (EE) for ATRA in NE was determined based on quantification of ATRA in the NE before (total ATRA) and after filtration (filtered ATRA; cellulose ester membrane, $0.45 \mu \mathrm{m}$, Millipore, Billerica, MA, USA). The fraction of ATRA soluble in the external AP of the NE was assessed by ultrafiltration, using a 100 $\mathrm{kDa}$ molecular weight cut-off membrane (Amicon $100 \mathrm{k}$, Millipore, Billerica, MA, USA).

ATRA concentration in NE (before and after filtration) or in the AP was determined by the differential pulse voltammetry (DPV) method reported by Silva and colleagues (Da Silva et al., 2015). Briefly, an aliquot of each NE formulation was dissolved in tetrahydrofuran and later diluted in a mixture (95:5) of methanol and 0.1 $\mathrm{M}$ acetate buffer ( $\mathrm{pH}$ 5.0). This dispersion was filtered in a $0.45 \mathrm{~mm}$ Millex HV filter (Millipore, Billerica, MA, USA) and analyzed by DPV. EE was then calculated using the following formula: $\mathrm{EE}(\%)=($ filtered ATRA/total ATRA $)$ X 100 .

The scan rate and pulse amplitude were $30 \mathrm{mV} \mathrm{s}^{-1}$ and $75 \mathrm{mV}$, respectively. The concentration range $(9.71-45.5$ $\left.\mathrm{x} 10^{-6} \mathrm{~mol} . \mathrm{L}^{-1}\right)$ used to determine the linear regression analysis resulted in the following linear equation: $\mathrm{y}=$ $0.03752 x+0.1717\left(R^{2}=0.9969\right)$.

\section{Stability study}

To investigate the influence of the coating on the NE stability, HA-ATRA-NE and ATRA-NE were prepared in triplicate, kept in $10 \mathrm{~mL}$ glass containers filled with a nitrogen atmosphere, and stored at $4{ }^{\circ} \mathrm{C}$. Sampling aliquots were taken at $7,15,30$, and 60 days of storage for mean diameter, zeta potential, $\mathrm{pH}$, and $\mathrm{EE}$ determinations.

\section{Polarized light microscopy}

The presence of ATRA crystals was assessed by polarized light microscopy (Zeiss Axio Imager.M2, Carl Zeiss, Oberkochen, Germany). Undiluted samples were placed on microscope slides, and images were acquired by utilizing a proper software (ZEN lite 2012, Carl Zeiss, Oberkochen, Germany). The microscope was equipped with an AxioCam digital camera (Model ERc 5S, Carl Zeiss, Oberkochen, Germany).

\section{Infrared analysis}

Fourier transform infrared (FTIR) measurements were carried out with a Nicolet 6700 spectrometer (Thermo Fisher Scientific, Waltham, USA) equipped with an attenuated total reflectance (ATR) accessory. Spectra were obtained using a Ge crystal in the region 
from 4000 to $700 \mathrm{~cm}^{-1}$ with a resolution of $4 \mathrm{~cm}^{-1}$ and 32 scan accumulations.

\section{In vitro release studies}

The in vitro release behavior of ATRA-NE and HA-ATRA-NE was determined in a dissolution medium composed of phosphate buffered saline ( $\mathrm{pH}$ 7.4): ethanol: Polysorbate 80 (88:10:2) using a dialysis method (Hu, Tang, Cui, 2004; Chinsriwongkul et al., 2012). $2 \mathrm{~mL}$ of formulation was applied in the dialysis bag with a 14 $\mathrm{kDa}$ cut-off (Sigma-Aldrich, USA). The dialysis bag was soaked in an amber flask containing $50 \mathrm{~mL}$ of dissolution medium under magnetic stirring of $100 \mathrm{rpm}$. Temperature was kept at $37 \pm 0.5^{\circ} \mathrm{C}$ and all samples were protected from light. At time intervals of 30 minutes, 1, 2, 4, 8, and 24 hours, $1 \mathrm{~mL}$ aliquot of the medium was taken, and the same volume of fresh medium was added. The amount of ATRA released to the medium at each time was quantified by DPV. All experiments were performed in triplicate.

\section{Cell cultures}

Cytotoxicity studies were conducted on cancer cells. Metastatic human breast adenocarcinoma cell lines (MCF-7 and MDA-MB-231) were cultivated in Dulbecco's modified Eagle's medium (DMEM) containing 10\% FBS, $200 \mathrm{mM}$ glutamine, and antibiotics (100 $\mu \mathrm{g} \mathrm{ml}^{-1}$ streptomycin and $100 \mathrm{UI} \mathrm{ml}^{-1}$ penicillin). All cultures were kept in a humidified incubator with 5\% $\mathrm{CO}_{2}$ at $37^{\circ} \mathrm{C}$.

\section{Analysis of cell viability}

Cell proliferation was measured by MTT assay as previously reported (Carneiro et al., 2012; Silva et al., 2015). First, $2.5 \times 10^{4}$ (MCF-7) or $2.0 \times 10^{4}$ (MDAMB-231) cells mL ${ }^{-1}$ were seeded into 96-well plates. After $24 \mathrm{~h}$ of incubation at $37^{\circ} \mathrm{C}$ and $5 \% \mathrm{CO}_{2}$, freshly prepared solutions of free ATRA and ATRA-loaded NE were added to the wells (ATRA concentration ranging from $1.56 \mu \mathrm{M}$ to $100 \mu \mathrm{M})$. Free ATRA was dissolved in absolute ethanol (4 mM) before dilution. Non-coated and HA-coated blank NE (without ATRA) were diluted in the same way as ATRA-loaded NE. After $48 \mathrm{~h}$ of incubation at $37^{\circ} \mathrm{C}$ and $5 \% \mathrm{CO}_{2}, 20 \mu \mathrm{L}$ of $5 \mathrm{mg} / \mathrm{mL}$ MTT solution was added to each plate. Plates were incubated at $37^{\circ} \mathrm{C}$ for $4 \mathrm{~h}$ and the medium was then replaced by $200 \mu \mathrm{L}$ of $0.04 \mathrm{~mol} \mathrm{~L}^{-1} \mathrm{HCl}$ solution in isopropanol. Cellular viability was estimated by measuring the rate of mitochondrial reduction of MTT into formazan of each treatment, determined by measuring the absorbance of the converted dye at a wavelength of $595 \mathrm{~nm}$, and compared with cells maintained in medium only ( $100 \%$ of viability). Cell viability was found to be $100 \%$ after treatment with negative control ( $2.5 \%$ ethanol). Data were expressed as percentage of cell viability in relation to the control (mean $\pm \mathrm{SD}$ ) for three independent experiments performed in sextuplicate.

\section{Data analysis}

Statistical analyses were carried out using one-way analysis of variance followed by Tukey's test. Differences among mean values were statistically significant when $P$ value was less than 0.05 . The statistical software used was GraphPad Prism 5.0 (Graphpad Software Inc., San Diego, USA).

\section{RESULTS AND DISCUSSION}

\section{Development of ATRA-NE formulations}

Blank NEs were developed and optimized by the spontaneous emulsification method. The physico-chemical parameters of the NE 1-5 including mean diameter, PDI, and zeta potential are summarized in Table II. Initially, the optimization of blank NE was investigated by evaluating the influence of surfactants on the globule size, PDI, and zeta potential. The presence of micrometric droplets increases the risk of embolism and the instability of NE, which is manifested by droplet coalescence. Thus, emulsions with monodisperse globules with size around 200-500 nm tend to be more stable and safer for intravenous administration (Hippalgaonkar, Majumdar, Kansara, 2010). Combination between polysorbate 80 and egg lecithin (NE 2, $346 \pm 15 \mathrm{~nm}$ ) caused a significant decrease in size when compared with their isolated use (NE 1, $422 \pm 9 \mathrm{~nm}$ ); both formulations were found to be monodisperse. The influence of cholesterol, a cosurfactant, was further investigated. Thus, NE 3 was prepared without cholesterol and again a significant decrease in the size was observed (NE 3, $280 \pm 1 \mathrm{~nm}$ ).

It is well known that globule size and PDI are strongly affected by the nature of the solvent used in the spontaneous emulsification process (Bouchemal et al., 2004). Then, ethanol was replaced by acetone in NE 4 , but due to the considerable increase in the size ( $424 \pm$ $14 \mathrm{~nm})$ and the high PDI $(0.42 \pm 0.049)$, and considering avoidance of potential toxic effects, ethanol was chosen as solvent of NE. Finally, NE 5 was prepared with the same composition and solvent of NE 3, but with a final stage of 10 minutes bath sonication, and a lower mean 
diameter $(135 \pm 2 \mathrm{~nm})$ and PDI $(0.17 \pm 0.016)$ were observed. Based on these findings, NE 5 was selected for the ATRA encapsulation studies. All blank formulations exhibited positive zeta potential due to the use of a cationic primary amine (SA) which remains at the interface of the NE globules. This positive charge is important to enable electrostatic attraction in the development of HA-coated NE formulations.

After ATRA incorporation (ATRA-NE), a significant decrease in mean particle size $(129 \pm 2 \mathrm{~nm})$ and a monodisperse distribution (PDI $0.18 \pm 0.005)$ were observed in comparison with the correspondent blank formulation (NE 5), as seen in Table II. Zeta potential was also properly positive $(35.7 \pm 1.0 \mathrm{mV})$. As expected, high EE $(96.7 \pm 4.9 \%)$ was obtained, which is in good agreement with our previous work involving incorporation of HIP between ATRA and lipophilic amines in lipid nanocarriers. ATRA concentration in the AP was found to be negligible as previously reported (Carneiro et al., 2012; Da Silva et al., 2015; Silva et al., 2015; Silva et al., 2016). The high ATRA retention in lipid matrix is related to the hydrophobicity of the counterion used in the HIP strategy, while the lipophilic amine is associated with intense interaction of ATRA with the lipid matrix (Oliveira et al., 2017). In this case, this formulation was considered suitable for the coating studies.

To determine the adequate $\mathrm{HA}$ concentration for $\mathrm{NE}$ coating, six HA concentrations from 0.10 to $4.0 \mathrm{mg} / \mathrm{mL}$ were tested. Considering that $\mathrm{pH}$ over the coating process was 7.0, both remaining SA (i.e., SA in excess, not in the ion pair with ATRA) and HA were ionized (pka of 10.21 and 4.36, respectively), which favored establishment of electrostatic interaction. As can be observed in Figure 1A, the increase of HA concentration led to a progressive increase in size and PDI, which can be explained by the coating deposition externally to the globules. Zeta potential was progressively reduced (Figure 1B), most likely due to the addition of the anionic HA molecules. The same reduction in zeta potential was observed by other reports (Yang et al., 2013; Tran et al., 2014) in the coating process with HA of paclitaxel and vorinostat lipid nanocarriers, respectively.

It was not possible to determine the physicochemical characteristics of HA-ATRA-NE coated with $0.25 \mathrm{mg} / \mathrm{mL}$ HA solution, since agglomeration was observed. The critical step in the electrostatic attraction coating process is the inversion of zeta potential, which indicates that the coating was successfully performed (Almalik, Day, Tirelli, 2013). However, it is possible for particle agglomeration to occur when its total charge becomes neutral.

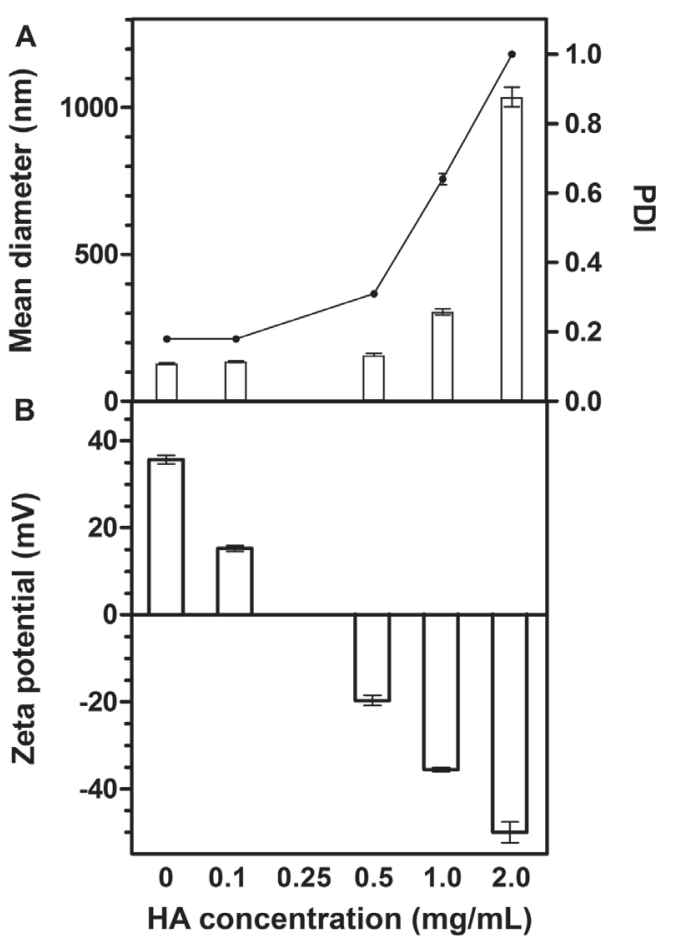

FIGURE 1 - Influence of HA concentration in mean globule diameter and PDI (A) and in zeta potential (B) of ATRA-loaded $\mathrm{NE}$ in the coating process.

The formulation selected for the further studies was HA-ATRA-NE coated by $0.5 \mathrm{mg} / \mathrm{mLHA}$, which presented the combination of small monodisperse globule size (158 \pm $5 \mathrm{~nm})$ and negative zeta potential $(-19.7 \pm 1.2 \mathrm{mV})$, suitable for the parenteral administration. Negative zeta potential is important to be achieved, since increased cytotoxicity is associated with positively charged nanoparticles and their interactions with the negatively charged cellular membrane (Lappalainen et al., 1994; Scholer et al., 2001; Carneiro et al., 2012).

In addition, ATRA kept its high encapsulation in the HA-coated NE $(99.2 \pm 0.5 \%)$, as can be seen in Figure 2. Likewise, drug loading remained unchanged ( $1.9 \%$ before and after the coating process). The concentration of ATRA was again found to be negligible in the AP. The presence of insoluble ATRA crystals in the external aqueous phase of ATRA-NE and HA-ATRA-NE was evaluated by polarized light microscopy in order to confirm the high EE. The obtained images are also shown in Figure 2, revealing that there were no ATRA crystals in the AP of both formulations, as expected (Carneiro et al., 2012; Silva et al., 2015; Silva et al., 2016).

\section{Infrared analysis}

HA-coated and non-coated formulations were 
TABLE II - Physicochemical characterization of NE formulations

\begin{tabular}{lccc}
\hline NE & $\begin{array}{c}\text { Diameter } \\
(\mathbf{n m})\end{array}$ & PDI & $\begin{array}{c}\text { Zeta Potential } \\
(\mathbf{m V})\end{array}$ \\
\hline 1 & $422 \pm 9$ & $0.18 \pm 0.006$ & $(+) 10.8 \pm 1.3$ \\
2 & $346 \pm 15$ & $0.22 \pm 0.007$ & $(+) 30.4 \pm 0.8$ \\
3 & $280 \pm 1$ & $0.19 \pm 0.003$ & $(+) 29.9 \pm 1.1$ \\
4 & $424 \pm 14$ & $0.42 \pm 0.049$ & $(+) 36.1 \pm 1.1$ \\
5 & $135 \pm 2$ & $0.17 \pm 0.016$ & $(+) 40.9 \pm 1.2$ \\
ATRA-NE & $129 \pm 2$ & $0.18 \pm 0.005$ & $(+) 35.7 \pm 1.0$ \\
\hline
\end{tabular}

Data represented as mean $\pm \mathrm{SD}(\mathrm{n}=3)$
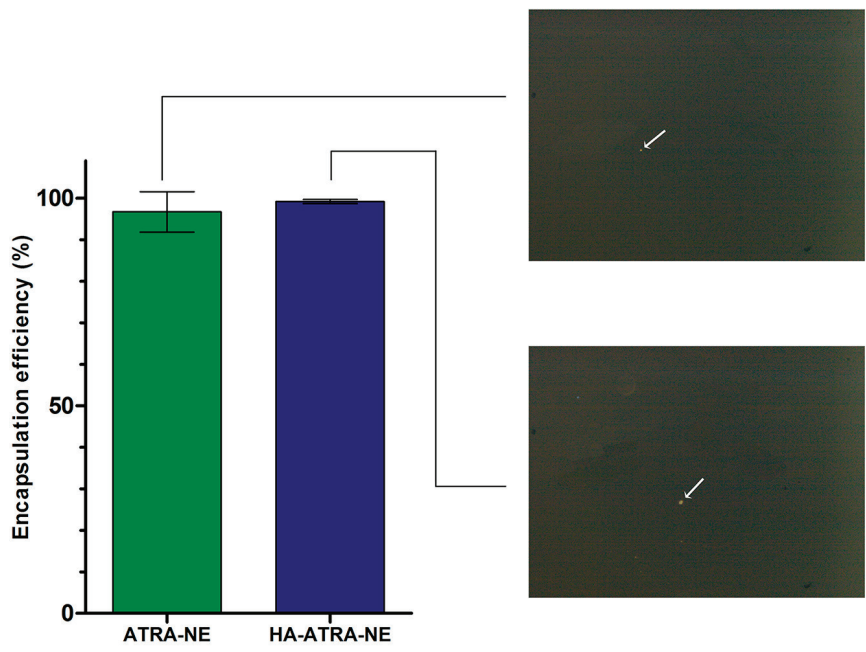

FIGURE 2 - Influence of HA coating on the encapsulation efficiency for ATRA in NE formulations. Data are shown as mean \pm SD. $n=3$. Rare ATRA microcrystals can be observed in the external phase of NE. indicating high encapsulation (Polarized Light Microscopy images. 40× magnification).

also investigated by ATR-FTIR (Figure 3) to confirm the coating process. Observed in the HA-ATRA-NE, in comparison with ATRA-NE, was a clear increase in the intensity of the band in $1745 \mathrm{~cm}^{-1}$ ascribed to carboxylic acid $\mathrm{C}=\mathrm{O}$ stretching mode and bands at 2850 and $2900 \mathrm{~cm}^{-1}$, related to $\mathrm{C}-\mathrm{H}$ stretching modes, suggesting the insertion of HA in the NE (Lopes et al., 2014). Corroborating with size and zeta potential data, FTIR results may provide additional evidence of the electrostatic attraction between the excess amine and the carboxylic group of HA, thus allowing coating of the globules (Tran et al., 2014).

\section{Stability studies}

After 60 days of storage in a refrigerator, no significant alteration in size was found for ATRA-NE

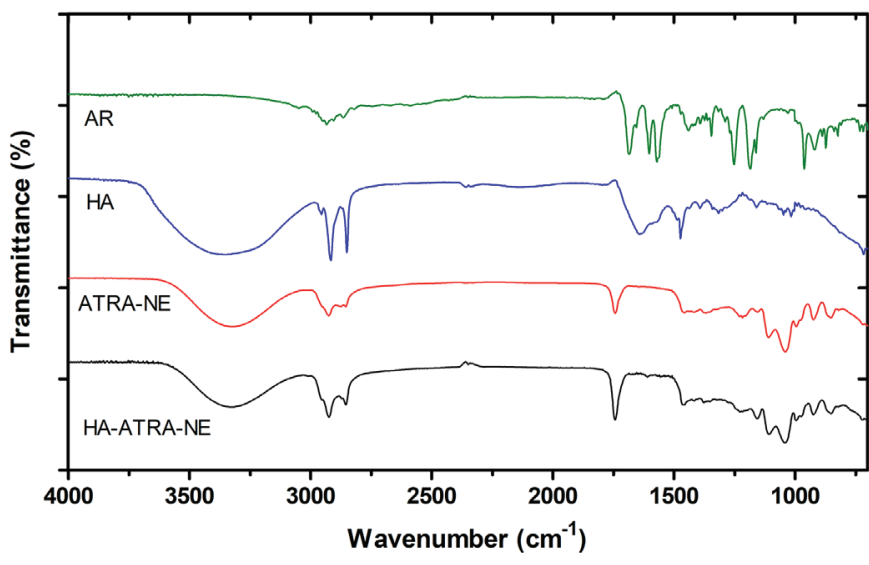

FIGURE 3 - FTIR-ATR spectra of ATRA, HA, coated and noncoated ATRA-loaded NE.

(Figure 4A), which was $169 \pm 0.6 \mathrm{~nm}$ after 60 days. PDI increased slightly, but the formulation was still considered monodisperse. HA-ATRA-NE had a significant increase in size from $140 \pm 2$ to $189 \pm 3 \mathrm{~nm}$ after 15 days storage, but remained constant up to 60 days (Figure 4B).

Zeta potential of both positively charged ATRA-NE and negatively charged HA-ATRA-NE remained constant over 60 days (Figure 4C). In addition, ATRA remained highly encapsulated in both coated and non-coated NE (EE were $96.5 \pm 1.0$ and $96.3 \pm 0.6 \%$, respectively, after 60 days). Finally, no presence of yellow crystals was observed at the external aqueous phase of both NE, indicating the high ATRA encapsulation over time.

\section{In vitro drug release}

Release profiles of ATRA from HA-coated and noncoated NE, and diffusion from ATRA solution are shown in Figure 5. In the same dissolution medium, the diffusion rate of ATRA solution was faster than that of the NE formulations. After 24 hours, $96.5 \pm 1.5 \%$ of free ATRA had already diffused against $90.1 \pm 1.1 \%$ (ATRA-NE) and $75.7 \pm 1.4 \%$ (HA-ATRA-NE). HA-ATRA-NE released ATRA in a significantly slower manner than ATRA-NE within 24 hours $(\mathrm{P}<0.05)$, indicating that the external hydrophilic HA coating could restrict the diffusion of ATRA through the encapsulating layer.

These results suggest that there is a controlled release of ATRA from HA-coated NE. In solution, drug release is a simple diffusion process from the dialysis bag to the receptor fluid; however, when encapsulated in a nanostructure, a mechanism that is slower to release the drug from the nanostructure core is usually needed, such as erosion, disintegration, or desorption (Rahman et al., 2013). 

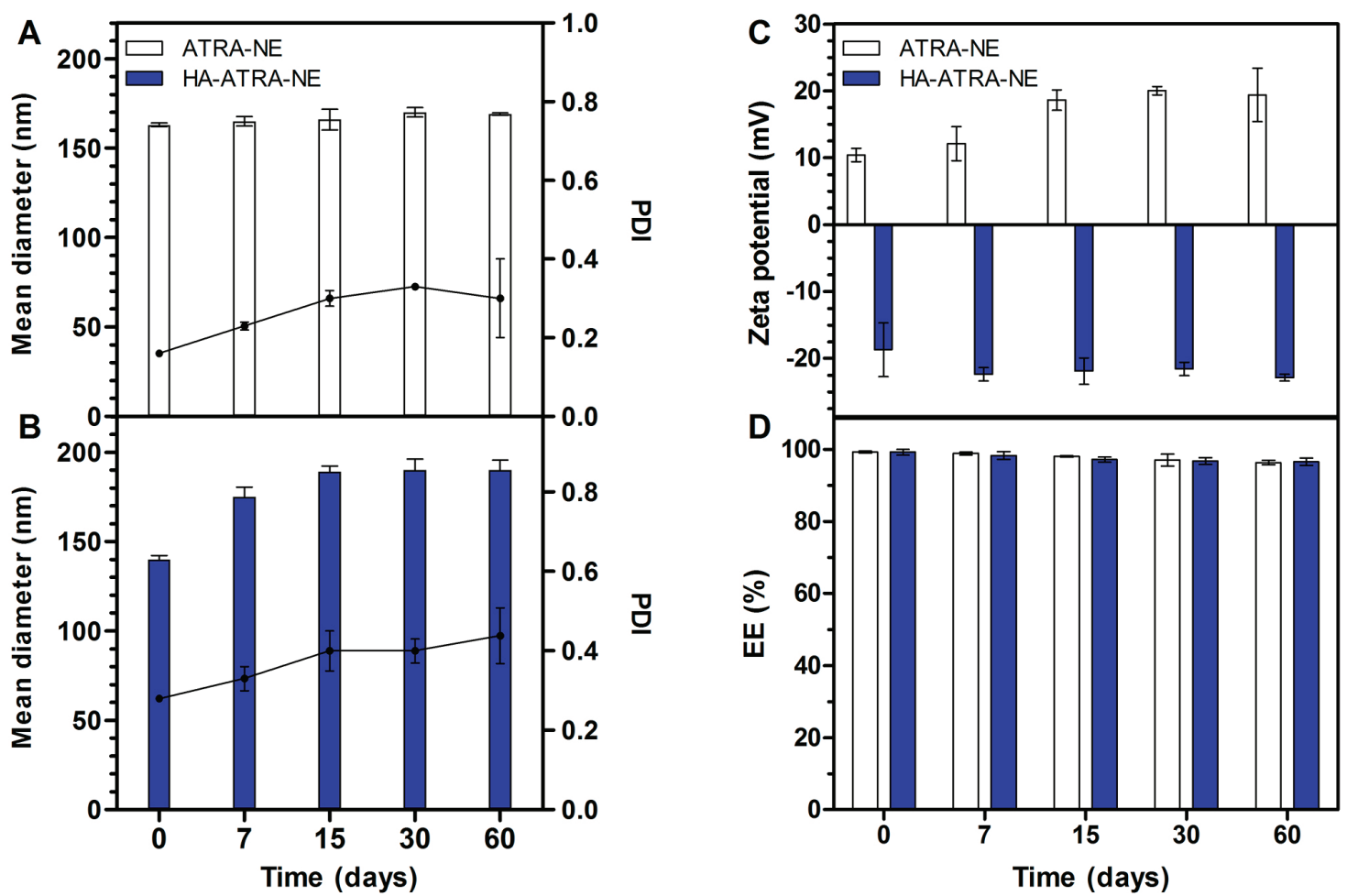

FIGURE 4 - Stability of mean diameter and PDI of ATRA-NE (A) and HA-ATRA-NE (B). Zeta potential (C) and encapsulation efficiency (D) of ATRA in NE. Data represented as mean $\pm \operatorname{SD}(n=3)$.

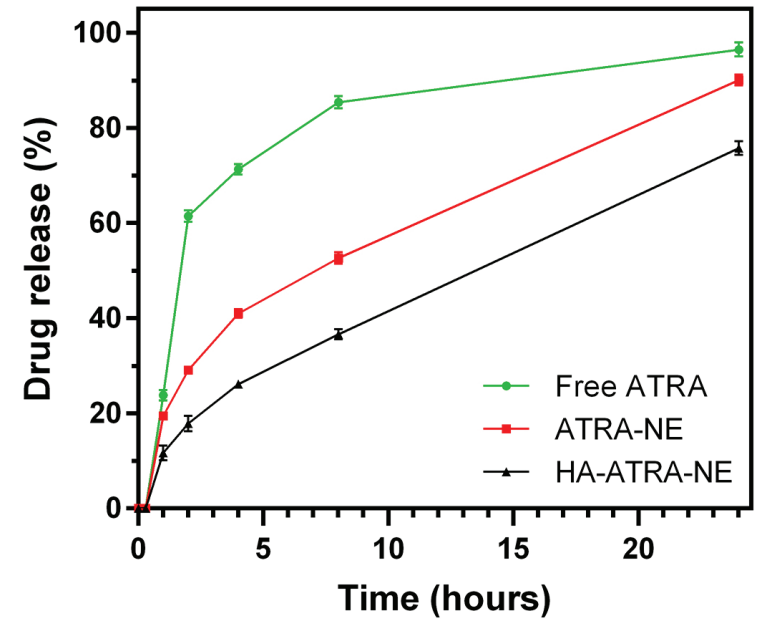

FIGURE 5 - Cumulative ATRA release from NE compared to a solution at $37 \pm 0.5^{\circ} \mathrm{C}$.

\section{Cell viability studies}

Breast cancer cell lines (MCF-7 and MBA-MB-231) were used to determine the cytotoxic activity of free ATRA or ATRA incorporated in the chosen HA-coated NE formulation. The cells were incubated with free ATRA or HA-ATRA-NE and analyzed with respect to their viability as shown in Figure 6. Cell viability was found to be $100 \%$ after treatment with negative control, and blank
HA-coated formulations exhibited little cytotoxicity (over $80 \%$ viability).

In all cell lines, there was a clear response to the treatment with free ATRA and the decrease in the cell viability was dose dependent, with a reduction from $100 \%$ at $1.56 \mu \mathrm{M}$ to $81 \pm 5.2 \%$ (MCF-7) and $86 \pm 2.1 \%$ at $50 \mu \mathrm{M}$ (MDA-MB-231), which are comparable to values obtained in previous studies (Fanjul et al., 1996; Hong, Lee-Kim, 2009).

Cytotoxic activity of ATRA incorporated in HAcoated NE on both cell lines was more pronounced than that of free ATRA. Cell viability was $33 \pm 2.8 \%$ in MCF7 cells and $24 \pm 1.6 \%$ in MDA-MB-231 cells at $50 \mu \mathrm{M}$. This effect could be associated with the lipophilic nature of the nanocarrier and enhanced intracellular absorption by CD44 receptor mediated endocytosis (Ramasamy et al., 2014; Tran et al., 2014). In addition, higher activity is expected for MDA-MB-231 cell lines, which is directly associated with CD44 receptor expression. Since MCF-7 cells express low levels of CD44 receptor (Tran et al., 2014), uptake of HA-coated NE would be lower when compared to MDA-MB-231 cells, with higher levels of CD44 expression. It is well-known that cationic SA formulations show cytotoxic effects associated with electrostatic interactions between the highly positive charged nanocarriers and anionic phospholipids of the 


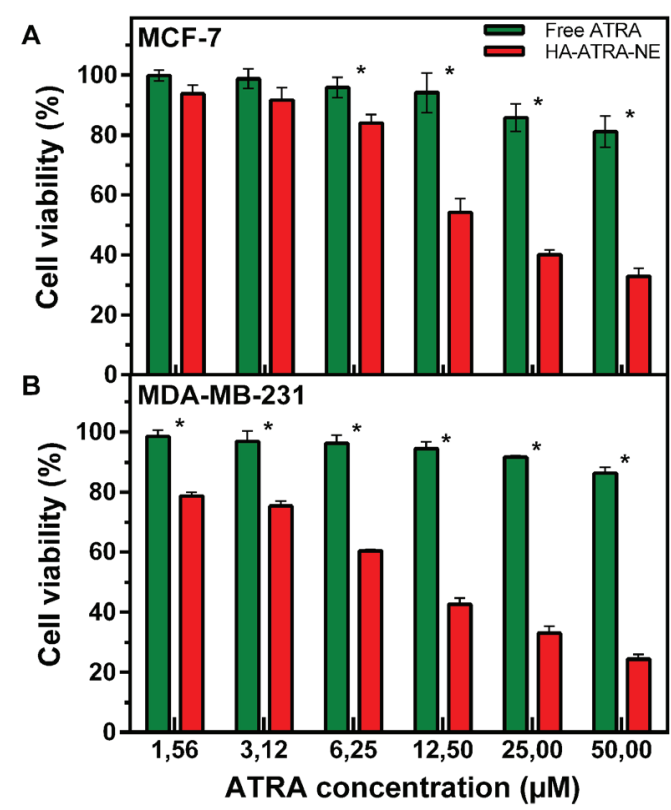

FIGURE 6 - Cell metabolic viability of MCF-7 (A) and MDAMB-231 (B) after 48 hours of exposure to blank NE, ATRAloaded NE, and free ATRA. Data represented as mean $\pm \mathrm{SD}$ $(n=3)$.

cell membrane, leading to damage of the latter (Roberts, Addy, 1981; Lappalainen et al., 1994; Scholer et al., 2001; Yang et al., 2013). In fact, this unspecific cytotoxicity was observed for unloaded SLN containing SA against cancer and normal cell lines (Lappalainen et al., 1994; Carneiro et al., 2012), which is not the case for blank HA-coated NE formulations.

\section{CONCLUSION}

A formulation of NE loaded with ATRA and coated with hyaluronic acid was developed with reduced size, negative zeta potential, and high encapsulation. This formulation was suitable for intravenous administration and remained stable over 60 days. ATRA was released more slowly from HA-coated NE compared to non-coated formulations, because of the additional hydrophilic layer of HA. HA-ATRA-NE was more cytotoxic against breast cancer cell lines than free ATRA, especially against cells overexpressing CD44.

\section{ACKNOWLEDGEMENTS}

This study was supported by Minas Gerais State Agency for Research and Development (FAPEMIG, Brazil) and by the Brazilian agencies CAPES and CNPq. This affiliation does not affect the conduct or reporting of this work submitted.

\section{REFERENCES}

Abdulmajed K, Heard CM. Topical delivery of retinyl ascorbate co-drug. 1. Synthesis, penetration into and permeation across human skin. Int J Pharm. 2004;280(1-2):113-124.

Adamson PC, Pitot HC, Balis FM, Rubin J, Murphy RF, Poplack DG. Variability in the oral bioavailability of all-trans-retinoic acid. J Natl Cancer Inst. 1993;85(12):993-996.

Almalik A, Day PJ, Tirelli N. HA-coated chitosan nanoparticles for CD44-Mediated nucleic acid delivery. Macromol Biosci. 2013;13(12):1671-1680.

Bouchemal K, Briancon S, Perrier E, Fessi H. Nano-emulsion formulation using spontaneous emulsification: solvent, oil and surfactant optimisation. Int J Pharm. 2004;280(1-2):241-251.

Carneiro G, Silva EL, Pacheco LA, de Souza-Fagundes EM, Correa NC, de Goes AM, et al. Formation of ion pairing as an alternative to improve encapsulation and anticancer activity of all-trans retinoic acid loaded in solid lipid nanoparticles. Int J Nanomedicine. 2012;7:6011-6020.

Castro GA, Coelho AL, Oliveira CA, Mahecha GA, Orefice RL, Ferreira LA. Formation of ion pairing as an alternative to improve encapsulation and stability and to reduce skin irritation of retinoic acid loaded in solid lipid nanoparticles. Int J Pharm. 2009;381(1):77-83.

Chansri N, Kawakami S, Yamashita F, Hashida M. Inhibition of liver metastasis by all-trans retinoic acid incorporated into $\mathrm{O} / \mathrm{W}$ emulsions in mice. Int J Pharm. 2006;321(1-2):42-49.

Chinsriwongkul A, Chareanputtakhun P, Ngawhirunpat T, Rojanarata T, Sila-on W, Ruktanonchai U, et al. Nanostructured lipid carriers (NLC) for parenteral delivery of an anticancer drug. AAPS PharmSciTech. 2012;13(1):150-158.

da Silva FLO, da Silva Tinoco LM, Ferreira LAM, Malagutti AR, Carneiro G. Determination of all-trans retinoic acid loaded in solid lipid nanoparticles by differential pulse voltammetry at glassy carbon electrode. Electrochim Acta. 2015;182:929-934.

di Masi A, Leboffe L, De Marinis E, Pagano F, Cicconi L, Rochette-Egly C, et al. Retinoic acid receptors: From molecular mechanisms to cancer therapy. Molecular Aspects Med. 2015;41:1-115. 
Fanjul AN, Bouterfa H, Dawson M, Pfahl M. Potential role for retinoic acid receptor-gamma in the inhibition of breast cancer cells by selective retinoids and interferons. Cancer Res. 1996;56(7):1571-1577.

Hippalgaonkar K, Majumdar S, Kansara V. Injectable lipid emulsions-advancements, opportunities and challenges. AAPS PharmSciTech. 2010;11(4):1526-1540.

Hong TK, Lee-Kim YC. Effects of retinoic acid isomers on apoptosis and enzymatic antioxidant system in human breast cancer cells. Nutr Res Pract. 2009;3(2):77-83.

Hu L, Tang X, Cui F. Solid lipid nanoparticles (SLNs) to improve oral bioavailability of poorly soluble drugs. J Pharm Pharmacol. 2004;56(12):1527-1535.

Hwang SR, Lim SJ, Park JS, Kim CK. Phospholipid-based microemulsion formulation of all-trans-retinoic acid for parenteral administration. Int J Pharm. 2004;276(1-2):175-183.

Jenning V, Gohla SH. Encapsulation of retinoids in solid lipid nanoparticles (SLN). J Microencapsul. 2001;18(2):149-158.

Kawakami S, Suzuki S, Yamashita F, Hashida M. Induction of apoptosis in A549 human lung cancer cells by all-trans retinoic acid incorporated in DOTAP/cholesterol liposomes. J Control Release. 2006;110(3):514-521.

Lappalainen K, Jaaskelainen I, Syrjanen K, Urtti A, Syrjanen S. Comparison of cell proliferation and toxicity assays using two cationic liposomes. Pharm Res. 1994;11(8):1127-1131.

Lim SJ, Kim CK. Formulation parameters determining the physicochemical characteristics of solid lipid nanoparticles loaded with all-trans retinoic acid. Int J Pharm. 2002;243(12):135-146.

Lim SJ, Lee MK, Kim CK. Altered chemical and biological activities of all-trans retinoic acid incorporated in solid lipid nanoparticle powders. J Control Release. 2004;100(1):53-61.

Lopes TD, Riegel-Vidotti IC, Grein A, Tischer CA, FariaTischer PC. Bacterial cellulose and hyaluronic acid hybrid membranes: Production and characterization. Int J Biol Macromol. 2014;67:401-408.

Mattheolabakis G, Milane L, Singh A, Amiji MM. Hyaluronic acid targeting of CD44 for cancer therapy: from receptor biology to nanomedicine. J Drug Target. 2015;23(7-8):605-618.
Muindi J, Frankel SR, Miller WH, Jr., Jakubowski A, Scheinberg DA, Young CW, et al. Continuous treatment with all-trans retinoic acid causes a progressive reduction in plasma drug concentrations: implications for relapse and retinoid "resistance" in patients with acute promyelocytic leukemia. Blood. 1992;79(2):299-303.

Oliveira MS, Goulart GC, Ferreira LA, Carneiro G. Hydrophobic ion pairing as a strategy to improve drug encapsulation into lipid nanocarriers for the cancer treatment. Expert Opin Drug Deliv. 2017;14(8):983-995.

Petrie K, Zelent A, Waxman S. Differentiation therapy of acute myeloid leukemia: past, present and future. Curr Opin Hematol. 2009;16(2):84-91.

Rahman HS, Rasedee A, How CW, Abdul AB, Zeenathul NA, Othman HH, et al. Zerumbone-loaded nanostructured lipid carriers: preparation, characterization, and antileukemic effect. Int J Nanomedicine. 2013;8:2769-2781.

Ramasamy T, Tran TH, Cho HJ, Kim JH, Kim YI, Jeon JY, et al. Chitosan-based polyelectrolyte complexes as potential nanoparticulate carriers: physicochemical and biological characterization. Pharm Res. 2014;31(5):1302-1314.

Rivkin I, Cohen K, Koffler J, Melikhov D, Peer D, Margalit R. Paclitaxel-clusters coated with hyaluronan as selective tumortargeted nanovectors. Biomaterials. 2010;31(27):7106-7114.

Roberts WR, Addy M. Comparison of the in vivo and in vitro antibacterial properties of antiseptic mouthrinses containing chlorhexidine, alexidine, cetyl pyridinium chloride and hexetidine. Relevance to mode of action. J Clin Periodontol. 1981;8(4):295-310.

Scholer N, Olbrich C, Tabatt K, Muller RH, Hahn H, Liesenfeld O. Surfactant, but not the size of solid lipid nanoparticles (SLN) influences viability and cytokine production of macrophages. Int J Pharm. 2001;221(1-2):57-67.

Silva EL, Carneiro G, Caetano PA, Goulart G, Ferreira Costa D, de Souza-Fagundes EM, et al. Nanostructured lipid carriers loaded with tributyrin as an alternative to improve anticancer activity of all-trans retinoic acid. Expert Rev Anticancer Ther. 2015;15(2):247-256. 
Silva EL, Lima FA, Carneiro G, Ramos Jonas P, Gomes DA, de Souza-Fagundes EM, et al. Improved in vitro antileukemic activity of all-trans retinoic acid loaded in cholesteryl butyrate solid lipid nanoparticles. J Nanosci Nanotechnol. 2016;16(2):1291-1300.

Sun H, Benjaminsen RV, Almdal K, Andresen TL. Hyaluronic acid immobilized polyacrylamide nanoparticle sensors for CD44 receptor targeting and $\mathrm{pH}$ measurement in cells. Bioconjug Chem. 2012;23(11):2247-2255.

Tang XH, Gudas LJ. Retinoids, retinoic acid receptors, and cancer. Annu Rev Pathol. 2011;6:345-364.
Torchilin V. Tumor delivery of macromolecular drugs based on the EPR effect. Adv Drug Deliv Rev. 2011;63(3):131-135.

Tran TH, Choi JY, Ramasamy T, Truong DH, Nguyen CN, Choi $\mathrm{HG}$, et al. Hyaluronic acid-coated solid lipid nanoparticles for targeted delivery of vorinostat to CD44 overexpressing cancer cells. Carbohydr Polym. 2014;114:407-415.

Yang X-y, Li Y-x, Li M, Zhang L, Feng L-x, Zhang N. Hyaluronic acid-coated nanostructured lipid carriers for targeting paclitaxel to cancer. Cancer Lett. 2013;334(2):338-345.

Received for publication on $29^{\text {th }}$ June 2017 Accepted for publication on $09^{\text {th }}$ March 2018 